\title{
IN VITRO ALPHA-GLUCOSIDASE INHIBITORY ACTIVITY OF EGYPTIAN PLANT EXTRACTS AS AN INDICATION FOR THEIR ANTIDIABETIC ACTIVITY
}

\author{
MAY A EL-MANAWATY*, LAMIAA GOHAR \\ Department of Pharmacognosy, Pharmaceutical and Drug Industries Research Division, National Research Centre, Cairo, Egypt. \\ Email: mayalyem@gmail.com
}

Received: 12 March 2018, Revised and Accepted: 16 April 2018

\begin{abstract}
Objective: Diabetes mellitus is a highly prevalent chronic disease in Egypt leading to high socioeconomic problems, especially in the cities due to the unhealthy life style. Although many drugs are available, they have many side effects. Furthermore, the body arouses resistance after a while for the drug so it should be changed every once in a while. Plants could be a good source for drugs. In Egypt, we have a rich flora which has not been subjected to systematic screening for antidiabetic activity.
\end{abstract}

Methods: The aim of this work was to screen 264 plant extracts for their in vitro $\alpha$-glucosidase inhibitory activity. Those extracts which gave more than $70 \%$ inhibition were screened on different concentrations and their inhibitory concentrations giving $50 \%$ activity $\left(\mathrm{IC}_{50}\right)$ were calculated.

Results: Out of all the tested extracts, 63 gave more than or equal $70 \%$ inhibition on $\alpha$-glucosidase at the tested concentration (25 ppm). After the calculation of the $\mathrm{IC}_{50}$ values, 10 extracts were chosen for further study having $5 \mathrm{ppm}$ and less $\mathrm{IC}_{50}$.

Conclusion: The most active plant extract is Pinus roxburghii Sarg. branches $\left(\mathrm{IC}_{50}\right.$ is $\left.2.47 \mathrm{ppm}\right)$.

Keywords: Antidiabetic, Alpha-glucosidase, In vitro, Plant extracts, Bioassay, Egyptian.

(C) 2018 The Authors. Published by Innovare Academic Sciences Pvt Ltd. This is an open access article under the CC BY license (http://creativecommons. org/licenses/by/4. 0/) DOI: http://dx.doi.org/10.22159/ajpcr.2018.v11i7.25856

\section{INTRODUCTION}

Diabetes is a complex metabolic condition where the patient suffers a chronic increase in blood glucose concentration (hyperglycemia) since the body cannot properly utilize it [1-4].

Diabetes mellitus is a highly prevalent chronic disease in Egypt leading to high socioeconomic problems, especially in the cities due to the unhealthy life style. Although many drugs are available, they have many side effects. Furthermore, the body arouses resistance after a while for the drug so it should be changed every once in a while.

It is noteworthy that therapeutic pathways for type 2 diabetes mellitus are based on the following: Stimulation of insulin secretion, reducing the production of glucose from the liver, interfering with the metabolism and digestion of carbohydrates (our target), and optimizing insulin action [5].

Plants and natural products could represent an excellent source for drugs since they can provide new compounds which cannot be predicted by computational chemistry [6].

The $\alpha$-glucosidase enzyme is responsible for changing oligo and disaccharides into monosaccharides. In case it is inhibited, the blood concentration of glucose decreases since only monosaccharides can be absorbed through intestinal mucosa [7] thus reducing the demand for insulin.

The aim of this work was to begin a series of diabetes-related in vitro screening program, studying the Egyptian flora. Since no systematic Egyptian large scale bio-study was done in that area before, we find it of great interest to undergo such a study.

The paper at hand, aimed to screen 264 plant extracts for their in vitro $\alpha$-glucosidase inhibitory activity.

\section{METHODS}

Preparation of the plant extracts

Seventy five grams of dried plant powder were percolated in $450 \mathrm{ml}$ 95\% analytical grade methanol (ADWIC-Egypt) for $24 \mathrm{~h}$ in a stainless steel percolator. The percolate was vacuum dried using Büchi R-114 rotatory evaporator at $45^{\circ} \mathrm{C}$. The distillate was used for re-percolation of the marc and the same procedure was adopted. The dried extract was kept in the dark at $-20^{\circ} \mathrm{C}$ till its use [8].

Preparation of the stock solutions of plant extracts for bioassays Ten mg of the dried methanol plant extract are dissolved in $500 \mu \mathrm{l}$ dimethyl sulfoxide (stock solution A). Master plates for the extracts to achieve different concentrations are prepared as follows: $5 \mu \mathrm{l}$ from the stock solution A + $195 \mu \mathrm{l}$ buffer (stock B); $100 \mu$ from the stock solution $\mathrm{B}+100 \mu \mathrm{l}$ buffer (stock C); $100 \mu \mathrm{l}$ from the stock solution $\mathrm{C}+100 \mu \mathrm{l}$ buffer (stock D); and $100 \mu \mathrm{l}$ from the stock solution C $+100 \mu \mathrm{l}$ buffer (stock D)

\section{$\alpha$-glucosidase inhibitory activity}

Briefly $\alpha$-glucosidase (Sigma G5003-100UN-USA) of concentration 0.2 $\mathrm{U} / \mathrm{ml}$ is prepared in phosphate buffer saline $(0.2 \mathrm{M})(\mathrm{pH} 6.8)(35.084 \mathrm{~g}$ $\mathrm{Na}_{2} \mathrm{HPO}_{4}$ [Loba Chemie-0597100500] + $13.872 \mathrm{~g} \mathrm{KH}_{2} \mathrm{PO}_{4}$ [s.d.fine-Chem ltd. BOISAR 39608]L). $10 \mu \mathrm{l}$ of sample at varying concentrations are mixed with $60 \mu \mathrm{l}$ of $0.2 \mathrm{U} / \mathrm{ml} \alpha$-glucosidase and incubated for $20 \mathrm{~min}$ at $37^{\circ} \mathrm{C}$ in a 96 well plate. The $150 \mu \mathrm{l}$ of $1.25 \mathrm{mM}$ p-nitrophenyl $\alpha$-Dglucopyranoside (p-NPG) (Sigma N1377-5g-USA) are added and incubated at $37^{\circ} \mathrm{C}$ for $20 \mathrm{~min}$ giving final concentrations of 0.2 to $25 \mathrm{ppm}$ in the final volume. The reaction is terminated by the addition of $50 \mu \mathrm{l}$ of $2 \mathrm{~g} / \mathrm{L}$ sodium hydroxide $(\mathrm{NaOH}) . \alpha$-glucosidase activity is determined spectrophotometrically at $405 \mathrm{~nm}$ by measuring the quantity of bright yellow p-nitrophenol released from the colorless p-NPG. The negative control has $10 \mu \mathrm{l}$ of buffer solution in place of the test entity while acarbose is used as a positive control. For blank p-NPG with buffer solution is added instead of the enzyme $[9,10]$. 
Calculation of the result:

The percentage inhibition $=(1-[$ average of sample absorbance/average of negative control absorbance] $) \times 100$

\section{RESULTS}

Two hundred and sixty four plant extracts (25 ppm) were screened for their $\alpha$-glucosidase inhibitory activity (Table 1 ).

Out of all the tested extracts, 63 gave more than or equal $70 \%$ inhibition on glucosidase at the tested concentration, where their $\mathrm{IC}_{50}$ values were calculated (Tables 2 and 3). IC $_{50}$ of acarbose as a positive control is $45 \pm 2.7 \mathrm{ppm}$. By reviewing the results, the most active plant extract proved to be Pinus roxburghii Sarg. branches methanol extract $\left(\mathrm{IC}_{50}\right.$ is $2.47 \mathrm{ppm})$.

\section{DISCUSSION}

The present study was designed for the purpose of screening a part of the Egyptian flora for their antidiabetic properties. Where a huge number of animals would be needed for the purpose, the resort to a simple in vitro method pointing out the plants which can be chosen as candidates for deeper in vivo studies is adopted. This paper is the beginning step in a much longer path so as to reach an effective antidiabetic drug from natural origin

By reviewing our findings, several plant extracts showed significant results in blocking the $\alpha$-glucosidase enzyme ability to break down starch, which, in turn, would decrease the blood glucose level.

Pinus roxburghii Sarg. being the most active plant was subjected to a literature survey. The survey showed that the bark alcohol extract has

Table 1: Screening of extracts on $\alpha$-glucosidase inhibitory in vitro bioassay at $25 \mathrm{ppm}$

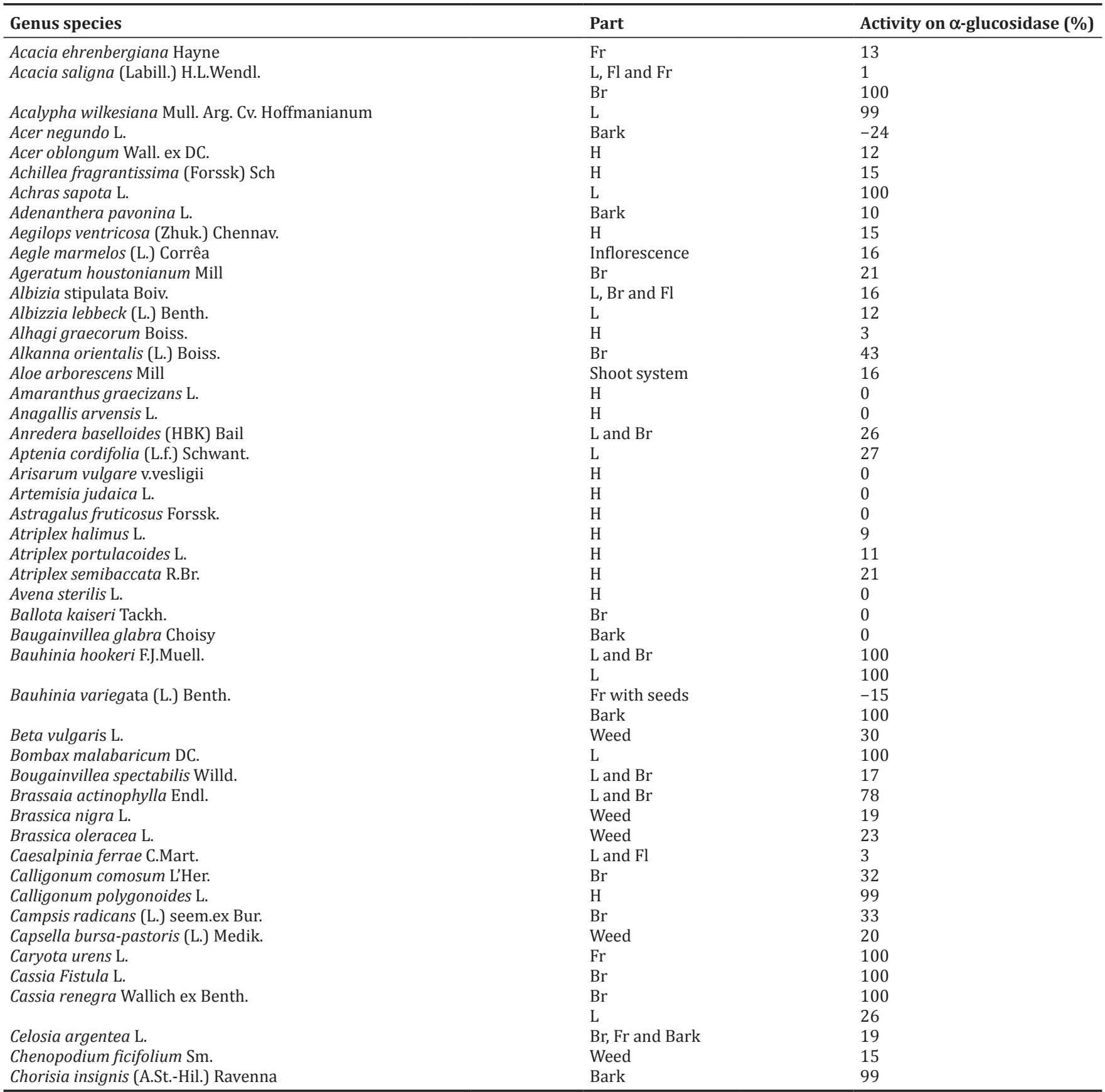


Table 1: (Continued)

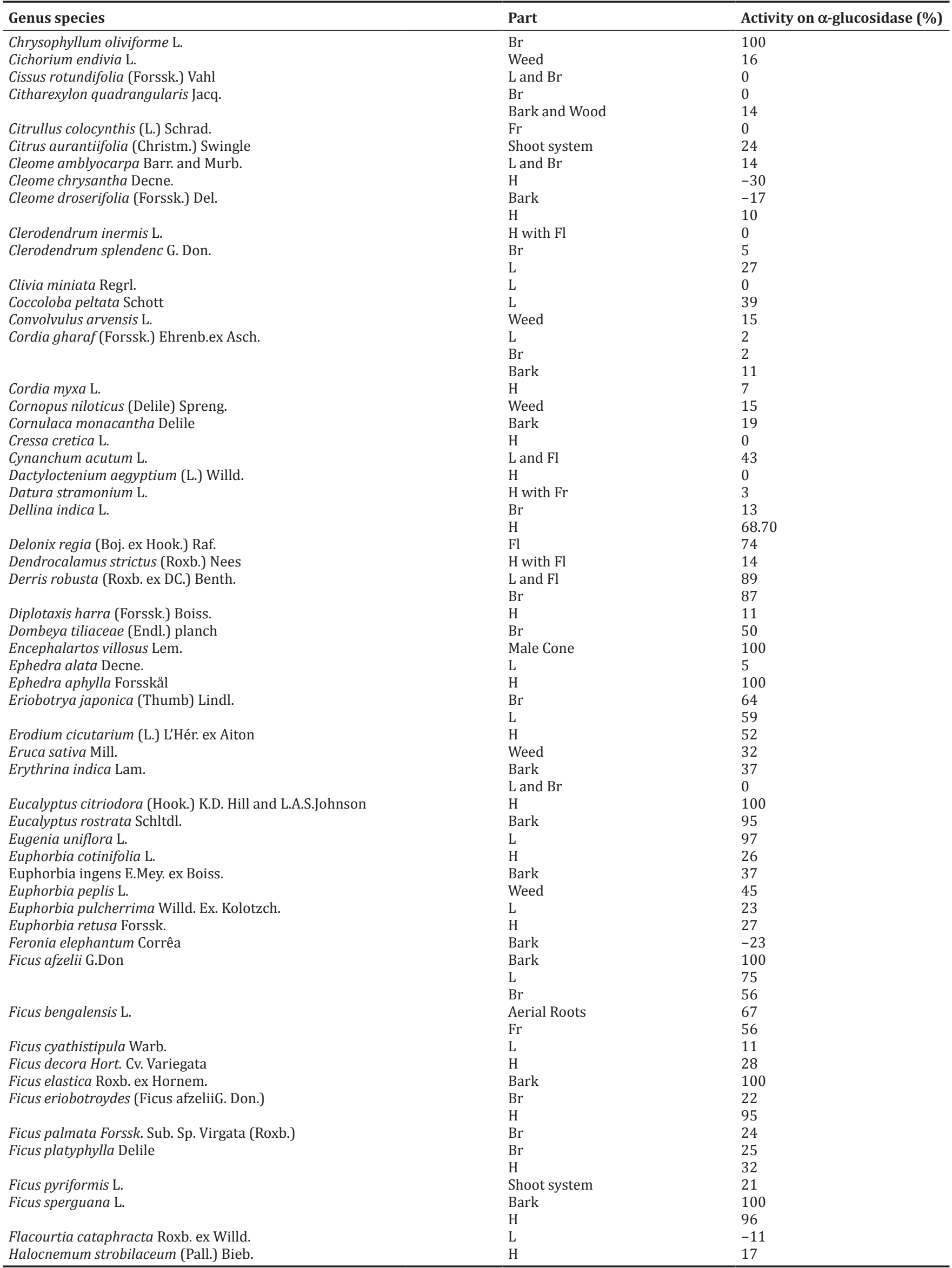


Table 1: (Continued)

\begin{tabular}{|c|c|c|}
\hline Genus species & Part & Activity on $\alpha$-glucosidase (\%) \\
\hline Harpephyllum caffrum Bernh. ex C.Krauss & $\mathrm{Br}$ & 6 \\
\hline \multirow[t]{4}{*}{ Harpullia cupanioides Roxb. } & Bark & 6 \\
\hline & $\mathrm{Br}$ & 83 \\
\hline & $\mathrm{L}$ & 0 \\
\hline & $\mathrm{H}$ & 15 \\
\hline Heliotropium digynum (Forssk.) Aschers. ex C. Christ. & $\mathrm{H}$ & -6 \\
\hline Hippeastrum vittatum (L Her.) & $\mathrm{Br}$ & 20 \\
\hline \multirow{2}{*}{ Hyphaene thebaica (L.) Mart. } & $\mathrm{Fl}$ & 2 \\
\hline & $\mathrm{H}$ & 0 \\
\hline Iphiona scabra DC. ex Decne. & $\mathrm{H}$ & 0 \\
\hline \multirow{2}{*}{ Jasminum primulinum Hemsl. } & $\mathrm{Br}$ & 18 \\
\hline & $\mathrm{L}$ & 33 \\
\hline Juncus rigidus Desf. & $\mathrm{H}$ & 0 \\
\hline \multirow{2}{*}{ Khaya dewi A.Juss. } & $\mathrm{L}$ and $\mathrm{Br}$ & 30 \\
\hline & $\mathrm{L}$ & 0 \\
\hline \multirow{2}{*}{ Khaya senegalensis (Desr.) A.Juss. } & $\mathrm{L}$ & 66 \\
\hline & $\mathrm{Br}$ & 83 \\
\hline Koelreuteria elegans (seem.) A.C, Sm.(Koelreuteria henryi & $\mathrm{L}$ & 96 \\
\hline \multicolumn{3}{|l|}{ Dummer) } \\
\hline Koelreuteria paniculata Laxm. & $\mathrm{Br}$ & 100 \\
\hline Lagerstroemia indica (L.) Pers. & $\mathrm{Br}$ & 87 \\
\hline Lamium amplexicaule $\mathrm{L}$. & Weed & 23 \\
\hline Lantana camara L. & $\mathrm{Br}$ & 0 \\
\hline Lasiurus hirsutus (Forssk.) Boiss. & $\mathrm{L}$ & 20 \\
\hline Launaea spinosa (Forssk.) Sch.Bip. & $\mathrm{H}$ & -5 \\
\hline \multirow[t]{2}{*}{ Leucaena glauca L. Benth } & $\mathrm{H}$ with $\mathrm{Fl}$ and $\mathrm{Fr}$ & 27 \\
\hline & $\mathrm{L}$ & 32 \\
\hline \multirow{2}{*}{ Liomoniastrum monopetalum (L.) Boiss. } & $\mathrm{L}, \mathrm{Br}$ and $\mathrm{Fl}$ & 8 \\
\hline & $\mathrm{H}$ & 100 \\
\hline Lonicera japonica Thunb. & $\mathrm{L}$ and $\mathrm{Br}$ & 23 \\
\hline Lotus tenuis Waldst. and Kit. ex Willd. & Weed & 16 \\
\hline \multicolumn{3}{|l|}{ Synonyms Lotus glaber Mill. } \\
\hline \multicolumn{3}{|l|}{ L. corniculatus subsp. tenuifolius L. } \\
\hline Maclura pomifera (Raf.) Schneid. & Bark & 0 \\
\hline \multirow{2}{*}{ Magnolia grandiflora L. } & Bark & -15 \\
\hline & $\mathrm{Fl}$ & 0 \\
\hline Malpighia glabra L. & $\mathrm{L}$ & 0 \\
\hline Malvaviscus arboreas Cav. & $\mathrm{H}$ & 21 \\
\hline Mangifera indica L. & $\mathrm{Br}$ & 96 \\
\hline Markhamia platycalyx (Baker) Sprague & $\mathrm{Fl}$ & 16 \\
\hline Medicago polymorpha $\mathrm{L}$. & Weed & 17 \\
\hline \multirow[t]{2}{*}{ Melaleuca ericifolia $\mathrm{Sm}}$. & $\mathrm{L}$ and $\mathrm{Fl}$ & 50 \\
\hline & $\mathrm{Br}$ & 55 \\
\hline Melia azedarach L. & Weed & 0 \\
\hline Melilotus indicus (L.) All. & Weed & 25 \\
\hline Mesembryanthemum nodiflorum L. & Weed & 19 \\
\hline Montanoa bipinnatifida (Kunth) K.Koch & $\mathrm{L}$ & -26 \\
\hline & $\mathrm{Br}$ & 2 \\
\hline Morettia philaena (Del.) DC & $\mathrm{Br}$ & 2 \\
\hline Morus alba L. & $\mathrm{L}$ and $\mathrm{Br}$ & 32 \\
\hline Morus nigra L. & Bark & 99 \\
\hline Myороrum laetum G. Forst. & $\mathrm{H}$ & 5 \\
\hline Nitraria retusa (Forssk.) Asch. & $\mathrm{H}$ & 0 \\
\hline Ononis vaginalis Vahl & $\mathrm{H}$ & 16 \\
\hline Oreopanax reticulatum Willd. & $\mathrm{Fr}$ & 0 \\
\hline Panicum turgidum Forssk. & $\mathrm{H}$ & 20 \\
\hline Parietaria judaica L. & $\mathrm{L}$ and $\mathrm{Br}$ & 30 \\
\hline Parkinsonia aculeata L. & $\mathrm{L}$ & 30 \\
\hline Paspalidium geminatum (Forssk.) Stapf. & $\mathrm{H}$ & 0 \\
\hline Phlomis aurea Decne. & Shoot system & 25 \\
\hline Phoenix dactylifera L. & Pulp & 0 \\
\hline Pinus canariensis C.Sm. & $\mathrm{Fr}$ & 100 \\
\hline & $\mathrm{L}$ & 100 \\
\hline Pinus halepensis Mill. & $\mathrm{Fr}$ & 75 \\
\hline & $\mathrm{Br}$ & 37 \\
\hline & $\mathrm{L}$ & 79 \\
\hline
\end{tabular}


Table 1: (Continued)

\begin{tabular}{|c|c|c|}
\hline Genus species & Part & Activity on $\alpha$-glucosidase (\%) \\
\hline & $\mathrm{L}$ & 99 \\
\hline \multirow[t]{3}{*}{ Pinus roxburghii Sarg. } & $\mathrm{Fr}$ & 30 \\
\hline & $\mathrm{Br}$ & 99.50 \\
\hline & $\mathrm{L}$ & 68 \\
\hline \multirow[t]{2}{*}{ Pistacia chinensis Bunge } & $\mathrm{L}$ and $\mathrm{Fr}$ & 11 \\
\hline & L and Unripe Fruit & 0 \\
\hline Pithecellobium dulce (Roxb.) Benth. & $\mathrm{Fr}$ & 100 \\
\hline Plumeria rubra L. & $\mathrm{L}, \mathrm{Br}$ and $\mathrm{Fr}$ & 15 \\
\hline Polyscias paniculata (DC.) Baker & $\mathrm{Br}$ & 100 \\
\hline \multirow[t]{2}{*}{ Psidium guajava $\mathrm{L}}$. & $\mathrm{Br}$ & 99 \\
\hline & $\mathrm{L}$ & 99 \\
\hline Pterospermum acerifolium (L.) Willd. & $\mathrm{Br}$ & 17 \\
\hline Pulicaria incisa (Lam.) DC. & H with Fl & 0 \\
\hline \multirow[t]{2}{*}{ Putranjiva roxburghii Wallich } & $\mathrm{Br}$ & 47 \\
\hline & $\mathrm{L}$ & 100 \\
\hline Pyrethrum santolinoides (Tanacetum santolinoides) & $\mathrm{H}$ & 0 \\
\hline Pyrus communis L. & Shoot system & 42 \\
\hline Ranunculus sceleratus L. & $\mathrm{H}$ & 0 \\
\hline Reseda pruinosa Delile & $\mathrm{H}$ & 9 \\
\hline Rumex dentatus $\mathrm{L}$. & Weed & 92 \\
\hline Sabal peregrina L.H. Bailey & $\mathrm{L}$ & 9 \\
\hline Salsola kali L. & $\mathrm{H}$ & 10 \\
\hline Sambucus nigra L. & Bark & 0 \\
\hline Saraca cauliflora Bak. & $\mathrm{L}$ & 91 \\
\hline Schefflera arboricola (Hayata) Kanehira & Shoot system & 30 \\
\hline Senecio glaucus L. Synonyms Senecio coronopifolius Desf & Weed & 23 \\
\hline Senna surattensis (Burm. f.) H. Irwin and Barneby & $\mathrm{Br}$ & 98 \\
\hline Sesbania sesban (L.) Merr. & $\mathrm{L}$ and $\mathrm{Br}$ & -17 \\
\hline Shinopsis balansea Engl & $\mathrm{Br}$ & 98 \\
\hline Silene rubella $\mathrm{L}$. & Weed & 19 \\
\hline Silybum marianum (L.) Gaertn. & Weed & 23 \\
\hline \multirow[t]{3}{*}{ Spondias lutea L. } & $\mathrm{L}$ & 89 \\
\hline & Fr & 100 \\
\hline & $\mathrm{Br}$ & 100 \\
\hline Stachys aegyptiaca Pers. & $\mathrm{H}$ & 0 \\
\hline Stellaria pallida (Dumort.) Piré & Weed & 34 \\
\hline \multirow[t]{2}{*}{ Sterculia foetida L. } & $\mathrm{L}$ & 51 \\
\hline & $\mathrm{Br}$ & 100 \\
\hline Suaeda aegyptiaca (Hasselq.) Zohary & $\mathrm{H}$ & 17 \\
\hline Suaeda pruinosa Lange & $\mathrm{H}$ & 32 \\
\hline Suaeda vera Forssk. ex J.F.Gmel. & $\mathrm{H}$ & 0 \\
\hline Swietenia macrophylla King & $\mathrm{L}$ & 62 \\
\hline \multirow[t]{3}{*}{ Swietenia mahagoni (L.) Jacq. } & $\mathrm{L}$ & 100 \\
\hline & $\mathrm{Br}$ & 100 \\
\hline & Bark & 42 \\
\hline Tecoma radicans (L.) Juss. & $\mathrm{L}$ and $\mathrm{Br}$ & 14 \\
\hline \multirow[t]{2}{*}{ Tecoma stans (L.) Juss. ex Kunth } & $\mathrm{H}$ & 21 \\
\hline & $\mathrm{L}$ & 26 \\
\hline Terminalia angustifolia Blanco & $\mathrm{Br}$ & 0 \\
\hline Terminalia arjuna (Roxb.) Wight and Arn. & $\mathrm{Br}$ & 100 \\
\hline Terminalia bellirica (Gaertn.) Roxb. & $\mathrm{Br}$ & 12 \\
\hline Teucrium polium L. & $\mathrm{H}$ & 0 \\
\hline \multirow[t]{3}{*}{ Tipuana tipu (Benth.) Kuntze } & Bark & 36 \\
\hline & $\mathrm{L}$ & 0 \\
\hline & $\mathrm{Fl}$ & 15 \\
\hline Trifolium resupinatum L. & Weed & 23 \\
\hline Trigonella stellata Forssk. & $\mathrm{H}$ & 0 \\
\hline Ulmus parvifolia Jacq. & $\mathrm{L}$ & 50 \\
\hline Verbascum sinaiticum Benth. & $\mathrm{Br}$ & 4 \\
\hline Verbena x hybrida Ross. & $\mathrm{L}$ & 15 \\
\hline Vicia sativa L. & Weed & 12 \\
\hline Woodfordia fruticose (L.) Kurz & Bark & 4 \\
\hline Xanthium spinosum L. & $\mathrm{H}$ & 0 \\
\hline Zilla spinosa (L.) Subsp. Parmata & $\mathrm{Br}$ & 12 \\
\hline
\end{tabular}

H: Herb, Fr: Fruits, Fl: Flowers, L: Leaves, Br: Branches 
Table 2: The percentage inhibition of extracts which gave $\geq 70 \%$ at $25 \mathrm{ppm}$ on $\alpha$-glucosidase on different concentrations to be used in the calculation of the IC values

\begin{tabular}{|c|c|c|c|c|c|c|c|}
\hline \multirow[t]{2}{*}{ Genus species } & \multirow[t]{2}{*}{ Part } & \multicolumn{6}{|c|}{ Activity on $\alpha$-glucosidase (\%) } \\
\hline & & 25 ppm & $12.5 \mathrm{ppm}$ & $6.3 \mathrm{ppm}$ & $3.1 \mathrm{ppm}$ & $1.6 \mathrm{ppm}$ & $0.8 \mathrm{ppm}$ \\
\hline Acacia saligna (Labill.) H.L. Wendl. & $\mathrm{Br}$ & 99 & 86 & 66 & 37 & & \\
\hline Acalypha wilkesiana Mull. Arg. Cv. Hoffmanianum & $\mathrm{L}$ & 99 & 95 & 71 & 30 & & \\
\hline Achras sapota $\mathrm{L}$ & $\mathrm{L}$ & 96 & 73 & 38 & 15 & & \\
\hline \multirow[t]{2}{*}{ Bauhinia hookeri F.J.Muell. } & $\mathrm{L}$ and $\mathrm{Br}$ & 90 & 88 & 73 & 34 & & \\
\hline & $\mathrm{Br}$ & 100 & 88 & 54 & 25 & & \\
\hline Bauhinia variegata (L.) Benth. & Bark & 71 & 71 & 59 & 54 & & \\
\hline Bombax malabaricum DC. & Bark & 97 & 78 & 48 & 24 & & \\
\hline Brassaia actinophylla Endl. & $\mathrm{L}, \mathrm{Br}$ & 83 & 64 & 33 & 15 & & \\
\hline Calligonum polygonoides L. & $\mathrm{H}$ & 100 & 100 & 97 & 50 & & \\
\hline Caryota urens L. & $\mathrm{L}$ & 99 & 90 & 60 & 27 & & \\
\hline Cassia Fistula L. & Bark & 95 & 82 & 59 & 40 & & \\
\hline Cassia renegra Wallich ex Benth. & $\mathrm{L}$ & 85 & 72 & 56 & 36 & & \\
\hline Chorisia insignis (A.St.-Hil.) Ravenna & Bark & 99 & 97 & 90 & 50 & & \\
\hline Chrysophyllum oliviforme $L$. & $\mathrm{Br}$ & 100 & 95 & 73 & 3 & & \\
\hline \multirow[t]{2}{*}{ Derris robusta (Roxb. ex DC.) Benth. } & $\mathrm{L}$ and $\mathrm{Fl}$ & 89 & 45 & 17 & 8 & & \\
\hline & $\mathrm{Br}$ & 88 & 82 & 79 & 46 & & \\
\hline Encephalartos villosus Lem. & Male Cone & 100 & 100 & 70 & 32 & & \\
\hline Ephedra aphylla Forsskål & Herb & 89 & 64 & 38 & 17 & & \\
\hline Eucalyptus citriodora (Hook.) K.D. Hill & Bark & 84 & 52 & 28 & 20 & & \\
\hline \multicolumn{8}{|l|}{ and L.A.S.Johnson } \\
\hline Eucalyptus rostrata Schltdl. & Bark & 95 & 87 & 81 & 73 & & \\
\hline Eugenia unifloraL. & $\mathrm{L}$ & 97 & 95 & 85 & 43 & & \\
\hline \multirow[t]{2}{*}{ Ficus afzelii G.Don } & Bark & 98 & 99 & 99 & 67 & 36 & 16 \\
\hline & $\mathrm{L}$ & 84 & 62 & 28 & 0 & & \\
\hline Ficus elastica Roxb. ex Hornem. & Bark & 100 & 96 & 87 & 40 & & \\
\hline Ficus eriobotroydes (Ficus afzeliiG. Don.) & $\mathrm{Br}$ and $\mathrm{Fr}$ & 88 & 51 & 41 & 2 & & \\
\hline \multirow[t]{2}{*}{ Ficus sperguana L. } & $\mathrm{L}$ & 83 & 62 & 42 & 20 & & \\
\hline & Bark & 100 & 98 & 73 & 12 & & \\
\hline \multirow[t]{2}{*}{ Harpullia cupanioidesRoxb. } & $\mathrm{Br}$ & 83 & 13 & 0 & & & \\
\hline & $\mathrm{Fl}$ & 72 & 39 & 21 & 10 & & \\
\hline \multirow[t]{2}{*}{ Khaya senegalensis (Desr.) A.Juss. } & $\mathrm{Br}$ & 86 & 81 & 51 & 13 & & \\
\hline & Bark & 99 & 98 & 98 & 76 & 45 & 34 \\
\hline \multirow{2}{*}{\multicolumn{8}{|c|}{ (Koelreuteria henryi Dummer) }} \\
\hline & & & & & & & \\
\hline Koelreuteria paniculata Laxm. & $\mathrm{Br}$ & 93 & 67 & 29 & 0 & & \\
\hline Lagerstroemia indica (L.) Pers. & $\mathrm{L}$ and $\mathrm{Fl}$ & 96 & 90 & 77 & 44 & & \\
\hline Liomoniastrum monopetalum (L.) Boiss. & Herb & 100 & 76 & 41 & 11 & & \\
\hline Mangifera indica $\mathrm{L}$. & $\mathrm{L}$ & 96 & 100 & 85 & 35 & & \\
\hline Morus nigra L. & Bark & 99 & 99 & 85 & 55 & & \\
\hline \multirow[t]{2}{*}{ Pinus canariensis C.Sm. } & $\mathrm{Fr}$ & 99 & 99 & 93 & 50 & & \\
\hline & $\mathrm{L}$ & 91 & 61 & 30 & 12 & & \\
\hline Pinus halepensis Mill. & $\mathrm{Fr}$ & 75 & 41 & 8 & 2 & & \\
\hline & $\mathrm{L}$ & 79 & 52 & 26 & 7 & & \\
\hline Pinus pinea $\mathrm{L}$. & $\mathrm{Br}$ & 92 & 57 & 0 & 0 & & \\
\hline & $\mathrm{L}$ & 91 & 62 & 26 & 4 & & \\
\hline Pinus roxburghii Sarg. & $\mathrm{Br}$ & 92.00 & 97 & 91 & 67 & & \\
\hline Pithecellobium dulce (Roxb.) Benth. & Bark & 95 & 94 & 78 & 38 & & \\
\hline polyscias paniculata (DC.) Baker & $\mathrm{Br}$ & 95 & 82 & 57 & 24 & & \\
\hline Psidium guajava L. & $\mathrm{Br}$ & 99 & 97 & 64 & 19 & & \\
\hline & $\mathrm{L}$ & 99 & 98 & 71 & 30 & & \\
\hline Putranjiva roxburghii Wallich & $\mathrm{L}$ & 87 & 54 & 37 & 11 & & \\
\hline Pyracantha fortuneana (Maxim) H.L.L.i & $\mathrm{B}$ & 98 & 94 & 70 & 47 & & \\
\hline (Pyracantha creneto-serrata (Hance Rehd) & & & & & & & \\
\hline Rumex dentatus L. & Weed & 75 & 38 & 10 & 4 & & \\
\hline Saraca cauliflora Bak. & $\mathrm{L}$ & 99 & 97 & 70 & 32 & & \\
\hline Senna surattensis (Burm. f.) H. Irwin and Barneby & $\mathrm{Br}$ & 99 & 89 & 64 & 27 & & \\
\hline Shinopsis balansea Engl & Bark & 98 & 97 & 77 & 41 & & \\
\hline Spondias lutea L. & $\mathrm{L}$ & 89 & 82 & 64 & 62 & & \\
\hline & $\mathrm{Br}$ & 100 & 99 & 88 & 51 & & \\
\hline & $\mathrm{Fr}$ & 66 & 37 & 0 & & & \\
\hline Sterculia foetida L. & $\mathrm{L}$ & 100 & 100 & 72 & 17 & & \\
\hline & $\mathrm{Br}$ & 100 & & 89 & 50 & & \\
\hline Swietenia mahagoni (L.) Jacq. & $\mathrm{L}$ & 99 & 98 & 88 & 52 & & \\
\hline & $\mathrm{Br}$ & 100 & 100 & 100 & 65 & & \\
\hline Terminalia arjuna (Roxb.) Wight and Arn. & $\mathrm{Br}$ & 100 & 100 & 60 & 30 & & \\
\hline
\end{tabular}

H: Herb, Fr: Fruits, Fl: Flowers, L: Leaves, Br: Branches 
Table 3: The calculated IC50 of the extracts which gave $\geq 70 \%$ at $25 \mathrm{ppm}$

\begin{tabular}{|c|c|c|}
\hline Plant name & Part & IC50 \\
\hline Acacia saligna (Labill.) H.L. Wendl. & $\mathrm{Br}$ & $05.64 \pm 1.0$ \\
\hline Acalypha wilkesiana Mull. Arg. Cv. Hoffmanianum & $\mathrm{L}$ & $05.43 \pm 0.9$ \\
\hline Achras sapota $\mathrm{L}$ & $\mathrm{L}$ & $09.17 \pm 1.7$ \\
\hline \multirow[t]{2}{*}{ Bauhinia hookeri F.J.Muell. } & $\mathrm{L}$ and $\mathrm{Br}$ & $06.20 \pm 1.6$ \\
\hline & $\mathrm{Br}$ & $06.26 \pm 0.9$ \\
\hline Bauhinia variegata (L.) Benth. & Bark & $07.95 \pm 1.3$ \\
\hline Bombax malabaricum DC. & Bark & $07.80 \pm 1.7$ \\
\hline Calligonum polygonoides L. & $\mathrm{H}$ & $03.54 \pm 0.5$ \\
\hline Caryota urens $\mathrm{L}$. & $\mathrm{L}$ & $11.63 \pm 2.1$ \\
\hline Cassia FistulaL. & Bark & $07.80 \pm 1.9$ \\
\hline Cassia renegra Wallich ex Benth. & $\mathrm{L}$ & $08.44 \pm 2.0$ \\
\hline Chorisia insignis (A.St.-Hil.) Ravenna & Bark & $02.97 \pm 0.8$ \\
\hline Chrysophyllum oliviforme L. & $\mathrm{Br}$ & $05.77 \pm 1.1$ \\
\hline \multirow[t]{2}{*}{ Derris robusta (Roxb. ex DC.) Benth. } & $\mathrm{L}, \mathrm{fl}$ & $18.83 \pm 2.7$ \\
\hline & $\mathrm{Br}$ & $05.53 \pm 1.6$ \\
\hline Eucalyptus citriodora (Hook.) K.D. Hill and L.A.S.Johnson & Bark & $12.41 \pm 2.1$ \\
\hline Eucalyptus rostrata Schltdl. & Bark & $03.20 \pm 0.1$ \\
\hline Eugenia uniflora L. & $\mathrm{L}$ & $04.36 \pm 0.8$ \\
\hline \multirow[t]{2}{*}{ Ficus afzelii G.Don } & Bark & $03.37 \pm 0.2$ \\
\hline & $\mathrm{L}$ & $13.23 \pm 1.1$ \\
\hline Ficus elastica Roxb. ex Hornem. & Bark & $03.99 \pm 0.1$ \\
\hline Ficus eriobotroydes (Ficus afzelii G. Don.) & $\mathrm{Br}$ and $\mathrm{Fr}$ & $13.18 \pm 1.4$ \\
\hline \multirow{2}{*}{ Ficus sperguana L. } & $\mathrm{L}$ & $11.66 \pm 2.1$ \\
\hline & Bark & $05.17 \pm 1.1$ \\
\hline \multirow[t]{2}{*}{ Harpullia cupanioides Roxb. } & $\mathrm{Br}$ & $21.01 \pm 4.1$ \\
\hline & $\mathrm{Fl}$ & $15.50 \pm 2.1$ \\
\hline \multirow[t]{2}{*}{ Khaya senegalensis (Desr.) A.Juss. } & $\mathrm{Br}$ & $08.21 \pm 1.7$ \\
\hline & Bark & $03.03 \pm 0.4$ \\
\hline Koelreuteria elegans (seem.) A.C, Sm. (Koelreuteria henryi & $\mathrm{L}$ & $03.96 \pm 0.3$ \\
\hline \multicolumn{3}{|l|}{ Dummer) } \\
\hline Koelreuteria paniculata Laxm. & $\mathrm{Br}$ & $11.18 \pm 2.0$ \\
\hline Morus nigra L. & Bark & $03.53 \pm 0.3$ \\
\hline \multirow[t]{2}{*}{ Pinus canariensis C.Sm. } & $\mathrm{Fr}$ & $04.37 \pm 0.7$ \\
\hline & $\mathrm{L}$ & $11.27 \pm 1.9$ \\
\hline \multirow[t]{2}{*}{ Pinus halepensis Mill. } & $\mathrm{Fr}$ & $17.00 \pm 3.1$ \\
\hline & $\mathrm{L}$ & $13.30 \pm 2.1$ \\
\hline \multirow[t]{2}{*}{ Pinus pinea $\mathrm{L}$. } & $\mathrm{Br}$ & $13.63 \pm 1.9$ \\
\hline & $\mathrm{L}$ & $12.07 \pm 2.0$ \\
\hline Pinus roxburghii Sarg. & $\mathrm{Br}$ & $02.47 \pm 0.2$ \\
\hline Pithecellobium dulce (Roxb.) Benth. & Bark & $05.22 \pm 1.0$ \\
\hline Polyscias paniculata (DC.) Baker & $\mathrm{Br}$ & $07.42 \pm 1.2$ \\
\hline \multirow[t]{2}{*}{ Psidium guajava $\mathrm{L}$. } & $\mathrm{Br}$ & $05.71 \pm 0.8$ \\
\hline & $\mathrm{L}$ & $04.99 \pm 0.2$ \\
\hline Putranjiva roxburghii Wallich & $\mathrm{L}$ & $11.97 \pm 2.1$ \\
\hline Pyracantha fortuneana (Maxim) H.L.L.i (Pyracantha & $\mathrm{Br}$ & $04.92 \pm 0.4$ \\
\hline \multicolumn{3}{|l|}{ creneto-serrata (Hance Rehd) } \\
\hline Rumex dentatus $\mathrm{L}$. & Weed & $17.60 \pm 2.4$ \\
\hline Saraca cauliflora Bak. & $\mathrm{L}$ & $05.29 \pm 0.8$ \\
\hline Senna surattensis (Burm. f.) H. Irwin and Barneby & $\mathrm{Br}$ & $06.43 \pm 0.1$ \\
\hline Shinopsis balansea Engl & Bark & $04.74 \pm 0.7$ \\
\hline \multirow{3}{*}{ Spondias lutea L. } & $\mathrm{L}$ & $05.30 \pm 0.5$ \\
\hline & $\mathrm{Br}$ & $03.72 \pm 0.3$ \\
\hline & $\mathrm{Fr}$ & $18.03 \pm 3.0$ \\
\hline \multirow[t]{2}{*}{ Sterculia foetida $\mathrm{L}}$. & $\mathrm{L}$ & $04.01 \pm 0.8$ \\
\hline & $\mathrm{Br}$ & $03.37 \pm 0.2$ \\
\hline \multirow{2}{*}{ Swietenia mahagoni (L.) Jacq. } & $\mathrm{L}$ & $03.58 \pm 0.3$ \\
\hline & $\mathrm{Br}$ & $02.64 \pm 0.1$ \\
\hline Terminalia arjuna (Roxb.) Wight and Arn. & $\mathrm{Br}$ & $04.92 \pm 1.2$ \\
\hline
\end{tabular}

H: Herb, Fr: Fruits, Fl: Flowers, L: Leaves, Br: Branches

anti-inflammatory and analgesic effect [11], the wood oil of the plant possesses hepatoprotective activity, and the essential oil of the needles is antibacterial and antifungal [12]. The antidiabetic in vivo activity of the bark extract and its hypolipidemic property were studied, where it proved to have a hypoglycemic effect [13]. The fore-mentioned result conforms to our work on the branches of the same plant. It was also studied as a protective for gastric ulcers [14] and for its antibacterial activity [15]. 


\section{CONCLUSION}

After the calculation of the IC values, 10 extracts were chosen having $5 \mathrm{ppm}$ and less $\mathrm{IC}_{50}$ which will be subjected to further extensive in vivo studies.

\section{ACKNOWLEDGMENT}

This work was financed by the National Research Centre, Egypt, under the project "Search of a novel anti-diabetic drug from natural origin;" contract agreement number 11010309 (2016-2019), Principal Investigator May A El-Manawaty.

\section{AUTHORS CONTRIBUTIONS}

Both authors contributed in the preparation of the extracts, the performance of the bioassay and the calculation of the $\mathrm{IC}_{50}$ values.

\section{CONFLICTS OF INTERESTS}

The authors have no conflicts of financial or personal interests with any other organizations or people.

\section{REFERENCES}

1. Alberti KG, Zimmet PF. Definition, diagnosis and classification of diabetes mellitus and its complications. Part 1: Diagnosis and classification of diabetes mellitus. Provisional report of a WHO consultation. Diabetic Med 1998;15:539-53.

2. Bhutkar M, Bhise S. In vitro hypoglycemic effects of Albizzia lebbeck and Mucuna pruriens. Asian Pac J Trop Biomed 2013;3:866-70.

3. Herman WH. Diabetes epidemiology: Guiding clinical and public health practice the kelly west award lecture. Diabetes Care 2007;30:1912-9.

4. Mellitus D. Diagnosis and classification of diabetes mellitus. Diabetes Care 2004;28:S37.

5. Sena CM, Bento CF, Pereira P, Marques F, Seiça R. Diabetes mellitus: New challenges and innovative therapies. In: New Strategies to Advance
Pre/Diabetes Care: Integrative Approach by PPPM. Netherlands: Springer; 2013. p. 29-87.

6. Uddin N, Hasan MR, Hossain MM, Sarker A, Hasan AN, Islam AM. Chowdhury MMH and Rana MS. In vitro $\alpha$-amylase inhibitory activity and in vivo hypoglycemic effect of methanol extract of Citrus macroptera Montr. fruit. Asian Pac J Trop Biomed 2014;4:473-9.

7. Funke I, Melzig MF. Traditionally used plants in diabetes therapyphytotherapeutics as inhibitors of $\alpha$-amylase activity. Rev Bras Farm 2006;16:1-5.

8. Fayad W, Salwa M, El-Manawaty MA, Mounier MM, Soliman AA, Yousry AA, et al. A systematic multicellular spheroids screening approach lead to the identification of antineoplastic activity in three different plant extracts from the Egyptian flora. J Appl Pharm Sci 2017;7:13-22

9. Elya B, Basah K, Yuliastuti AW, Bangun A, Septiana EK. Screening of $\alpha$-glucosidase inhibitory activity from some plants of Apocynaceae, Clusiaceae, Euphorbiaceae, and Rubiaceae. J Biomed Biotechnol 2012;2012:1-6.

10. Qaisar MN, Chaudhary BA, Sajid MU, Hussain N. Evaluation of $\alpha$-glucosidase inhibitory activity of dichloromethane and methanol extracts of Croton bonplandianum Baill. Trop J Pharm Res 2014;13:1833-6.

11. Kaushik D, Kumar A, Kaushik P, Rana AC. Analgesic and antiinflammatory activity of Pinus roxburghii Sarg. Adv Pharmacol Sci 2012;2012:1-6.

12. Kaushik P, Kaushik D, Khokra SL. Ethnobotany and phytopharmacology of Pinus roxburghii Sargent: A plant review. J Integr Med 2013;11:371-6.

13. Kaushik P, Khokra SL, Kaushik D. Evaluation of antidiabetic potential of Pinus roxburghii bark extract in alloxan induced diabetic rats. J Pharm Nat Prod 2015;1:1-5.

14. Chaudhary AK, Ahmad S, Mazumder A. Protective effect of Cedrus deodara and Pinus roxburghii on experimentally induced gastric ulcers in rat. Int J Pharm Pharm Sci 2014;6:587-91.

15. Rana IS, Singh A, Gwal R. In vitro study of antibacterial activity of aromatic and medicinal plants essential oils with special reference to cinnamon oil. Int J Pharm Pharm Sci 2011;3:376-80. 